\title{
Cytologic Characteristics of Thymic Adenocarcinoma with Enteric Differentiation: A Study of Four Fine-Needle Aspiration Specimens
}

\author{
Ah-Young Kwon · Joungho Han \\ Hae-yon Cho · Seokhwi Kim \\ Heejin Bang · Jiyeon Hyeon \\ Department of Pathology, Samsung Medical \\ Center, Sungkyunkwan University School of \\ Medicine, Seoul, Korea \\ Received: February 17, 2017 \\ Revised: March 14, 2017 \\ Accepted: March 22, 2017 \\ Corresponding Author \\ Joungho Han, MD \\ Department of Pathology, Samsung Medical Center, \\ Sungkyunkwan University School of Medicine, 81 \\ Irwon-ro, Gangnam-gu, Seoul 06351, Korea \\ Tel: +82-2-3410-2765 \\ Fax: +82-2-3410-0025 \\ E-mail: hanjho@skku.edu
}

\begin{abstract}
Thymic adenocarcinoma is extremely rare. Although its histologic features have been occasionally reported, a lack of description of the cytologic features has hampered the prompt and accurate diagnosis of this condition. Herein, we describe the cytologic findings and histology of four aspiration cytology specimens of thymic adenocarcinoma. The specimens were obtained from primary tumors, metastatic lymph nodes, and pericardial effusions. All four specimens showed threedimensional glandular clusters with a loss of polarity and nuclear overlapping. One specimen had extensive extracellular mucinous material. Three specimens contained tumor cells with intracytoplasmic vacuoles. While the specimen with extracellular mucin showed relatively mild cytologic atypia, other specimens exhibited more atypical cytologic changes: irregular nuclear membranes, a coarse chromatin pattern, and prominent nucleoli. The cytologic features were correlated with the histologic features in each case of enteric type thymic adenocarcinoma. The differential diagnosis included other thymic carcinomas, yolk sac tumors, and metastatic adenocarcinoma from the lung or colorectum.
\end{abstract}

Key Words: Mediastinum; Thymus gland; Adenocarcinoma; Cytology; Aspiration
Primary thymic adenocarcinoma is a very rare neoplasm that accounts for $0.48 \%$ of all thymic epithelial tumors. ${ }^{1}$ Only 58 cases have been reported to date. ${ }^{2}$ Based on histology, thymic adenocarcinoma is classified into four subtypes: mucinous, papillary, not otherwise specified (NOS), and a type with adenoid cystic carcinoma-like features. ${ }^{3}$ Recently, Moser et al. ${ }^{4}$ and our institute ${ }^{2}$ reported that both mucinous and NOS types showed enteric differentiation, suggesting that enteric type thymic adenocarcinoma could be an appropriate term in regards to the histology of this condition.

Previous studies have focused on the diagnosis and classification of thymic adenocarcinoma according to its clinical, histologic, and immunohistochemical features. However, the cytologic features of thymic adenocarcinoma have not yet been reported. In this study, we investigated the characteristics of thymic adenocarcinoma in aspiration cytology based on four specimens from three patients. The clinical and histologic features of these specimens were reported in our previous study. ${ }^{2}$ We analyzed the cytologic features and histologic characteristics as well as the differential diagnosis of thymic adenocarcinoma.

\section{CASE REPORT}

The clinicopathologic characteristics of four thymic adenocarcinoma specimens are summarized in Table 1 . These four specimens were obtained from three patients, who were reported in our previous study ${ }^{2}$ as cases 6,7 , and 8 . All cytology specimens were obtained by fine-needle aspiration (FNA) from the subaortic and right supraclavicular lymph nodes, pericardial effusion, and tumors. This study was approved by the Institutional Review Board of Samsung Medical Center (IRB file No. 2016-07-064), and informed consent was waived.

\section{Patient 1 (specimen 1)}

A 70-year-old man visited Samsung Medical Center complaining of chest wall pain. Chest computed tomography (CT) revealed a 9.6-cm-sized cystic lesion in the anterior and left upper mediastinum with direct extension to the mediastinal fat and the 
Table 1. Clinicopathologic characteristics of cases

\begin{tabular}{|c|c|c|c|c|c|c|c|c|}
\hline $\begin{array}{l}\text { Case } \\
\text { No. }\end{array}$ & Sex & $\begin{array}{l}\text { Age } \\
\text { (yr) }\end{array}$ & $\begin{array}{l}\text { Smoking } \\
\text { history }\end{array}$ & Past history & $\begin{array}{l}\text { Masaoka } \\
\text { stage }\end{array}$ & $\begin{array}{l}\text { Specimen } \\
\text { of aspiration }\end{array}$ & Cytologic diagnosis & Histologic diagnosis \\
\hline 1 & M & 70 & $40 \mathrm{PY}$ & HTN, DM & $\|$ & $\begin{array}{r}\text { Mediastinal LN } \\
\text { (subaortic LN) }\end{array}$ & $\begin{array}{l}\text { Metastatic carcinoma, showing } \\
\text { glandular clusters of atypical } \\
\text { cells }\end{array}$ & Thymic adenocarcinoma, enteric type \\
\hline \multirow[t]{2}{*}{2} & M & 50 & $20 \mathrm{PY}$ & Angina & IVB & Supraclavicular LN & $\begin{array}{l}\text { Metastatic carcinoma, showing } \\
\text { glandular clusters of atypical } \\
\text { cells }\end{array}$ & Thymic adenocarcinoma, enteric type \\
\hline & & & & & & Pericardial effusion & $\begin{array}{l}\text { Metastatic carcinoma, showing } \\
\text { glandular clusters of atypical } \\
\text { cells }\end{array}$ & - \\
\hline 3 & M & 62 & $5 \mathrm{PY}$ & HTN, DM & IVB & Tumor & $\begin{array}{l}\text { A few clusters of atypical cells } \\
\text { in mucinous background }\end{array}$ & $\begin{array}{l}\text { Thymic adenocarcinoma, enteric } \\
\text { mucinous type }\end{array}$ \\
\hline
\end{tabular}

M, male; PY, pack-year; HTN, hypertension; DM, diabetes mellitus; LN, lymph node.

left upper lobe of the lung. Multiple enlarged lymph nodes were found in the mediastinum. The patient underwent endobronchial ultrasound (EBUS)-transbronchial needle aspiration (TBNA) of the subaortic lymph node. FNA slides revealed some epithelial clusters in bloody background (Fig. 1A). Three-dimensional (3D) clusters consisted of variably sized cells with moderate to high nuclear atypia. Their nuclei had irregular contours, margination, a coarse chromatin pattern, and single or two prominent nucleoli. Cytoplasmic borders were indistinct, and the amount of cytoplasm was variable (Fig. 1B). Some of the tumor cells contained intracytoplasmic vacuoles (Fig. 1A, arrow). Core needle biopsy (CNB) was conducted a day after the EBUS-TBNA. The tumor was diagnosed as thymic adenocarcinoma with enteric differentiation (Fig. 1C). The histologic characteristics were well correlated with the features identified in cytologic specimens: a tubulo-glandular pattern with nuclear atypia and occasional intracytoplasmic mucin.

\section{Patient 2 (specimens 2 and 3)}

A 50-year-old man visited the hospital for an evaluation of his weight loss ( $8 \mathrm{~kg}$ during the past two months). Chest CT revealed an 8.1-cm-sized, heterogeneously enhancing mass in the anterior mediastinum with sternum destruction. Multiple small nodules in the lung parenchyma and necrotic lymphadenopathy in the right supraclavicular area were also identified. FNA of the right supraclavicular lymph node revealed a large amount of three-dimensional epithelial cell clusters in a background of blood and inflammatory cells (Fig. 1D, E). The cells at the edge of the clusters were stratified. Small to large clusters consisted of columnar cells with abundant eosinophilic cytoplasm and occasional intracytoplasmic vacuoles (Fig. 1F). The elongated nuclei of the columnar cells had irregular membranes with margination, coarse to vesicular chromatin, and prominent nucleoli. $\mathrm{CNB}$ for the mediastinal mass was subsequently performed. A tubular growth pattern, pseudostratified nuclei with atypia, and a few instances of intracytoplasmic mucin were also found in the CNB specimen (Fig. 1I). Considering the cytology and histology of the tumor, enteric type adenocarcinoma was an appropriate diagnosis.

After 6 months of prolonged chemotherapeutic treatment, pericardial effusion from the patient was obtained by aspiration. Liquid-based cytology showed crowded epithelial cells with nuclear overlapping (Fig. 1G). Pleomorphic nuclei, vesicular chromatin, and single or two prominent nucleoli were also shown (Fig. 1H).

\section{Patient 3 (specimen 4)}

A 62-year-old man presented to the hospital with chest discomfort. Chest CT showed an infiltrative anterior mediastinal mass, measuring $3.2 \mathrm{~cm}$ in size, along with massive pericardial effusion. The possibility of lymphoma or thymic malignancy was suggested considering the patient's clinical and radiologic features. FNA of the anterior mediastinum was conducted. In the aspirated specimen, a few cells could be identified with extensive mucinous material in the background (Fig. 1J). Tumor cells were round and small to medium in size. A relatively fine chromatin pattern and indistinct nucleoli favored benign cells; however, slight nuclear margination, irregular arrangement, and cellular overlapping suggested malignancy (Fig. 1K). The patient underwent surgical excision. According to the cytologic and histologic features of the specimen, a diagnosis of enteric type mucinous adenocarcinoma was made (Fig. 1L). One major histologic characteristic of the surgical specimen was floating tumor clusters in the extracellular mucin that were cribriform, tubular, or single cell types.

\section{DISCUSSION}

Thymic adenocarcinoma is rarely encountered in routine practice. However, with the increasing frequency of EBUS, pathologists 

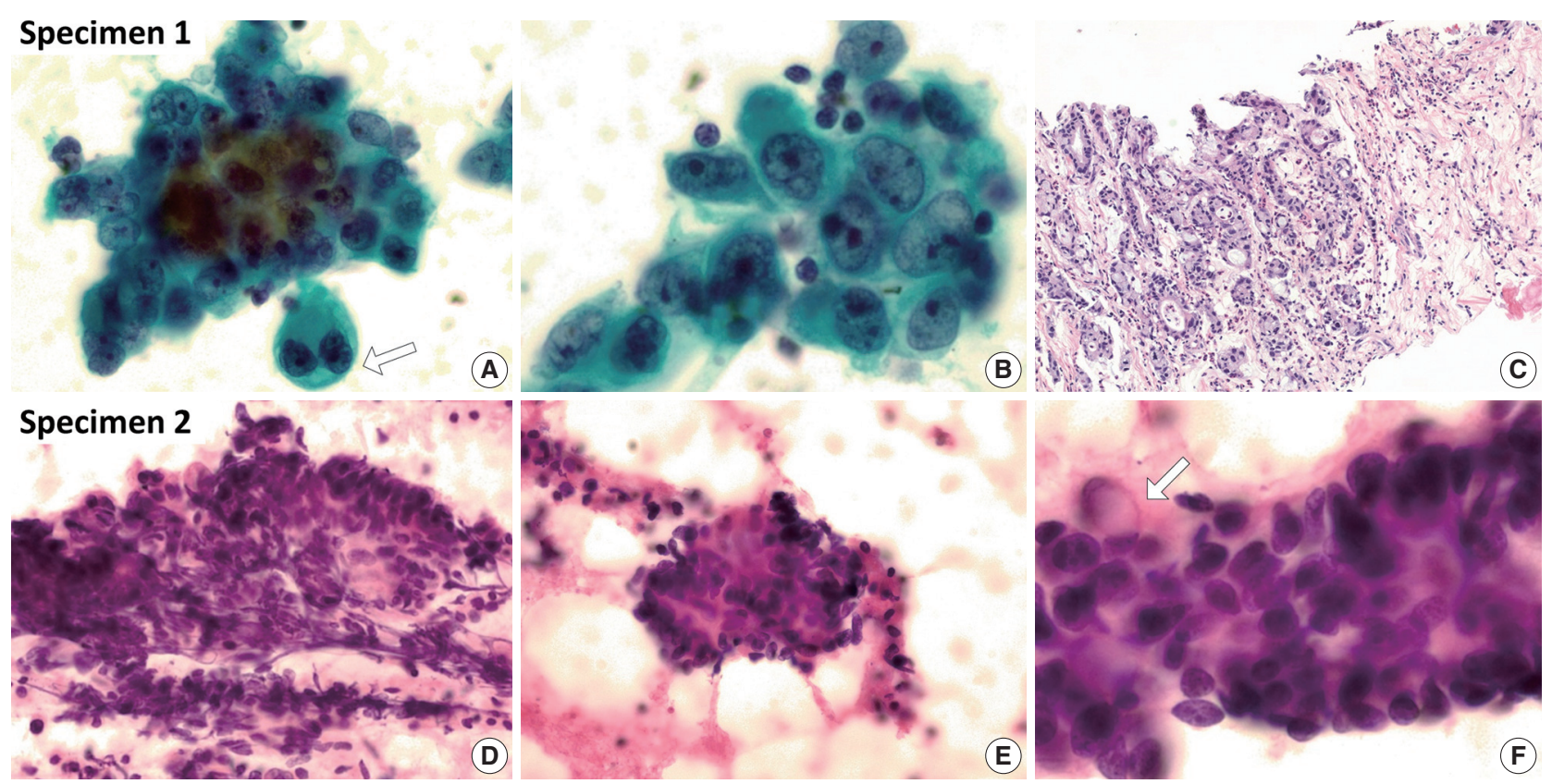

Specimen 3
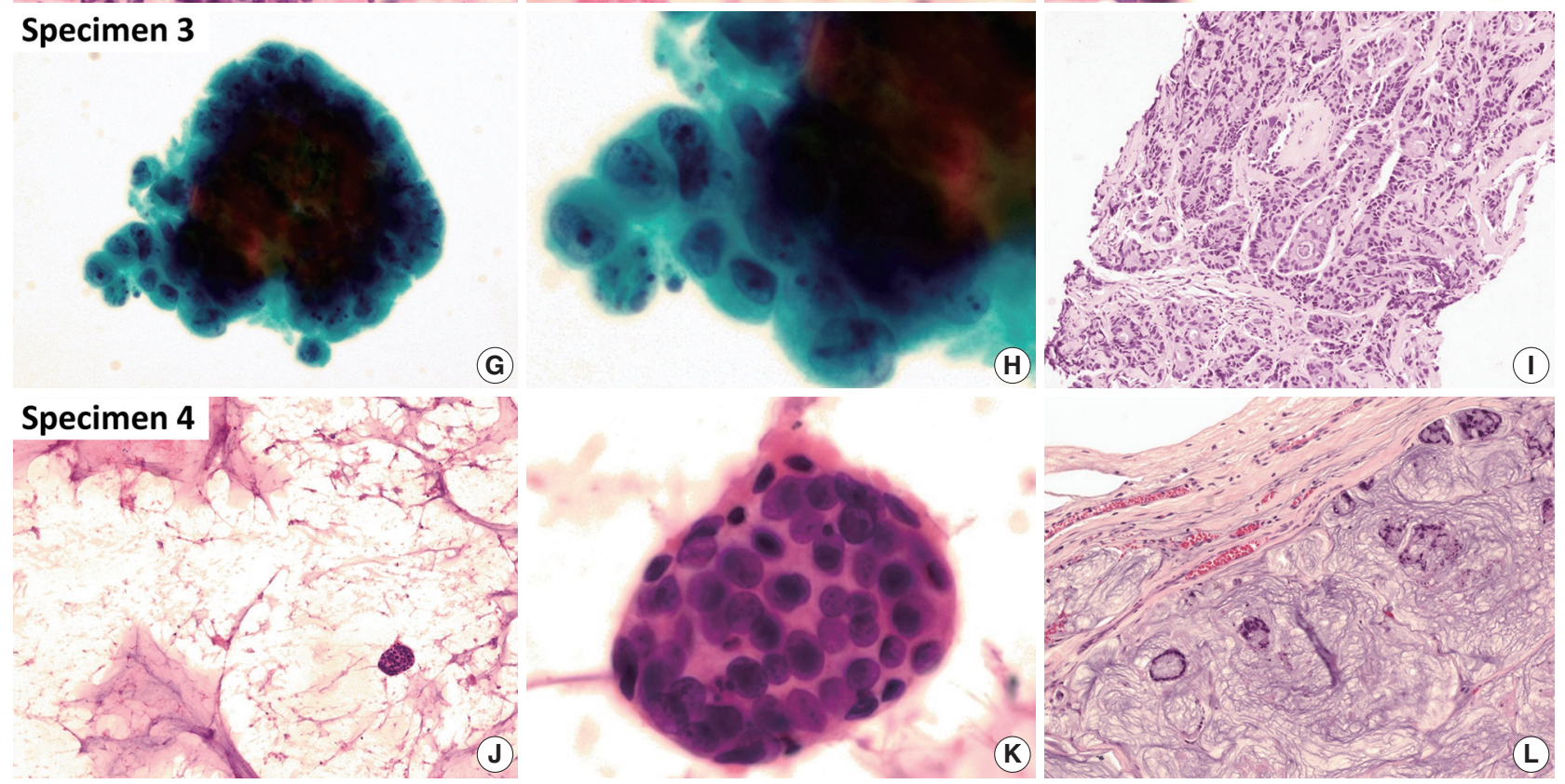

Fig. 1. Cytologic characteristics of thymic adenocarcinoma with histology. (A-C) The fine-needle aspiration (FNA) specimen was obtained from a mediastinal lymph node. Some epithelial cell clusters with a three-dimensional glandular structure are shown. The variably-sized cells have atypical nuclei showing an irregular margin, distinct margination, vesicular chromatin, and prominent nucleoli. Loss of polarity is also present. The cytoplasm is moderately abundant, and intracytoplasmic vacuoles are not infrequently seen (arrow). Adenocarcinoma with a tubular pattern is revealed in the histomorphology of the core biopsy from the tumor. Glandular structure, atypical nuclei, eosinophilic cytoplasm, intracytoplasmic vacuoles, and surrounding inflammatory cells and fibrotic stroma are identical findings with aspiration cytology (A and B, WrightGiemsa stain). (D-I) Two separate FNAs were carried out in a supraclavicular lymph node (D-F) and the pericardial effusion (G, H). There are glandular- and cribriform-patterned clusters with an inflammatory background. Palisading and stratification are frequently found in clusters. An irregular nuclear margin, vesicular chromatin, prominent nucleoli, and an occasional intracytoplasmic vacuole (arrow) are shown in this sample from patient 2. A core biopsy specimen was obtained from the mediastinal tumor. The tumor shows a tubular and cribriform pattern, stratified nuclei, eosinophilic cytoplasm, intracytoplasmic vacuoles, and inflammatory cells (G and H, Wright-Giemsa stain). (J-L) Most of the aspiration specimen obtained from the tumor is mucinous material. Only a few clusters can be detected. A round cluster of tumor cells reveals relatively mild nuclear atypia. Nuclear overlapping and irregular arrangement are still observed. The excisional specimen reveals floating tumor cells with a tubular or cribriform pattern within the extensive extracellular mucin. 
should be aware of the cytologic features of thymic adenocarcinoma in aspiration. In addition, thymic adenocarcinomas often accompany metastasis at the time of diagnosis. Therefore, the importance of an accurate diagnosis of aspiration cytology in mediastinal tumors, lymph nodes, or effusion is increasing.

Thymic adenocarcinoma may be classified into four categories based on histology. We previously reported that mucinous adenocarcinoma and adenocarcinoma NOS could be referred to as enteric type adenocarcinoma. ${ }^{2}$ This enteric type is the most common; all 14 cases reported in Korea have been of this type. ${ }^{2}$ Although some individual reports have mentioned the diagnosis of thymic adenocarcinoma in aspiration specimens, ${ }^{5-7}$ a detailed description of the cytologic diagnosis of thymic adenocarcinoma has not been reported to date. ${ }^{2,8-12}$

In our FNA specimens, thymic adenocarcinoma cells demonstrated 3D clusters with nuclear crowding and overlapping. Other cytologic findings were similar to histomorphology. In specimens 1 and 2, the columnar cells had stratified nuclei and occasional intracytoplasmic vacuoles. The nuclei had the following signs, which suggest malignancy: irregularity of the nuclear membrane, nuclear margination, coarse chromatin, and prominent nucleoli. A dirty background containing blood and inflammatory cells could aid in a confirmative diagnosis. Malignant features of the nuclei were not clear for the mucinous type (specimen 4). However, extensive extracellular mucin and loss-of-polarity of clusters were characteristically identified. Their cytologic features were more prominent when compared with histologic specimens.

The differential diagnosis of thymic adenocarcinoma includes thymic epithelial malignancies, direct invasion of pulmonary adenocarcinoma, metastatic adenocarcinoma, and germ cell tumors, especially yolk sac tumors. Aspiration cytology of yolk sac tumors might show similar cytologic features to thymic adenocarcinoma, such as atypical cells with a glandular pattern and a dirty background. The serum $\alpha$-fetoprotein level and immunohistochemical staining could be helpful in the differential diagnosis. Other thymic carcinomas might reveal epithelial cell clusters with nuclear atypia and crowding. Glandular tumor clusters and intracellular or extracellular mucin favor a diagnosis of adenocarcinoma. Metastatic adenocarcinoma from other organs should be regarded as an important differential diagnosis. In particular, colonic adenocarcinoma and pulmonary enteric type adenocarcinoma might be difficult to differentiate from primary thymic adenocarcinoma because their histologic features and immunohistochemical profile are very similar. Therefore, clinical and radiologic findings such as location, size, and the presence of metastasis of the tumor should be rigorously analyzed and carefully considered.

\section{Conflicts of Interest}

No potential conflict of interest relevant to this article was reported.

\section{REFERENCES}

1. Ahmad U, Yao X, Detterbeck F, et al. Thymic carcinoma outcomes and prognosis: results of an international analysis. J Thorac Cardiovasc Surg 2015; 149: 95-100.

2. Kwon AY, Han J, Chu J, et al. Histologic characteristics of thymic adenocarcinomas: clinicopathologic study of a nine-case series and a review of the literature. Pathol Res Pract 2017; 213: 106-12.

3. Marx A, Chan JK, Coindre JM, et al. The 2015 World Health Organization classification of tumors of the thymus: continuity and changes. J Thorac Oncol 2015; 10: 1383-95.

4. Moser B, Schiefer AI, Janik S, et al. Adenocarcinoma of the thymus, enteric type: report of 2 cases, and proposal for a novel subtype of thymic carcinoma. Am J Surg Pathol 2015; 39: 541-8.

5. Banki F, Khalil K, Kott MM, Cota AL. Adenoid cystic carcinoma of the thymus gland: a rare tumor. Ann Thorac Surg 2010; 90: e56-8.

6. Furtado A, Nogueira R, Ferreira D, Tente D, Eisele R, Parente B. Papillary adenocarcinoma of the thymus: case report and review of the literature. Int J Surg Pathol 2010; 18: 530-3.

7. Zaitlin N, Rozenman J, Yellin A. Papillary adenocarcinoma in a thymic cyst: a pitfall of thoracoscopic excision. Ann Thorac Surg 2003; 76: 1279-81.

8. Abdul-Ghafar J, Yong SJ, Kwon W, Park IH, Jung SH. Primary thymic mucinous adenocarcinoma: a case report. Korean J Pathol 2012; 46: 377-81.

9. Cho EN, Park HS, Kim TH, et al. A rare case of primary thymic adenocarcinoma mimicking small cell lung cancer. Tuberc Respir Dis 2015; 78: 112-9.

10. Jung HY, Cho H, Chung JH, et al. A rare case of primary tubular adenocarcinoma of the thymus, enteric immunophenotype: a case study and review of the literature. J Pathol Transl Med 2015; 49: 331-4.

11. Ra YJ, Bae MJ, Kim YS, Choi KU. Difficulties in diagnosis and treatment of thymic adenocarcinoma producing beta-human chorionic gonadotropin in anterior mediastinum. Interact Cardiovasc Thorac Surg 2010; 11: 114-6.

12. Seon HJ, Kim KH, Choi YD, et al. Angina pectoris caused by the extrinsic compression of coronary artery by primary thymic mucinous adenocarcinoma. Int J Cardiol 2012; 156: e13-5. 